\title{
PRODUÇÃO CIENTÍFICA SOBRE A CLASSIFICAÇÃO INTERNACIONAL DAS PRÁTICAS DE ENFERMAGEM EM SAÚDE COLETIVA
}

\author{
Simone Karine da Costa MESQUITA* \\ Déborah Karollyne Ribeiro RAMOS** \\ *Enfermeira. Mestre em Enfermagem pela Universidade Federal do Rio Grande do Norte. Especialista em Saúde da \\ Família. Enfermeira de Unidade Básica de Saúde/RN. e-mail: simone.karine@ @otmail.com \\ **Enfermeiro. Mestre em Enfermagem pela Universidade Federal do Rio Grande do Norte. Especialista em Saúde \\ Mental. Docente da Faculdade de Ciências Médicas de Campina Grande. E-mail: Deborah.ribeiro.ramos@gmail.com
}

Recebido em: 29/05/2014 - Aprovado em: 18/09/2014 - Disponibilizado em: 15/12/2014

\begin{abstract}
Resumo
A Classificação Internacional das Práticas de Enfermagem em Saúde Coletiva - CIPESC é um instrumento do processo de trabalho assistencial do enfermeiro em saúde coletiva que favorece a organização do trabalho, trazendo valorização, benefícios e impactos da ação da enfermagem tanto na assistência quanto na gerencia e pesquisa. Diante disto, o presente estudo teve como objetivo analisar a produção científica acerca da classificação internacional das práticas de enfermagem em saúde coletiva. Trata-se de uma pesquisa de revisão integrativa, realizada por meio do levantamento de artigos em periódicos da Biblioteca Virtual em Saúde (BVS), indexados na base de dados SciELO, LILACS, MEDLINE e BDENF, utilizando como descritor "Enfermagem em saúde pública", e a palavra-chave "CIPESC". O universo a pesquisa foi constituído por 60 artigos, contudo 14 artigos fizeram parte da amostra. Pesquisou-se publicações completas e online entre 2000 e 2012. De acordo com os resultados da pesquisa pode-se evidenciar que o método descritivo-exploratório teve uma maior representabilidade nos artigos analisados. Em $72 \%$ dos artigo, não ocorreram a utilização de teorias nas pesquisas por parte dos pesquisadores. Referente a ano de publicação, o ano de 2007 apresentou maior destaque. Contudo, vale ressaltar que a utilização do referido instrumento aprimora as práticas de enfermagem, trazendo visibilidade e autonomia, favorecendo um modo de pensar consciente e sistemático no âmbito da prática de saúde coletiva.
\end{abstract}

Palavras-chave: Enfermagem em saúde pública. Classificação. CIPESC. Enfermagem. Sáude Coletiva.

\begin{abstract}
The International Classification of Nursing Practice in Public Health - CIPESC is an instrument of the working process of the attending nurse in public health and favors the organization of work, bringing value, benefits and impacts of nursing action either for assistance in managing and research. Hence, the present study aimed to analyze the scientific production concerning the international classification of nursing practice in public health. It is an integrative review of research conducted by the survey papers in the Virtual Health Library ( VHL ), indexed in SciELO, LILACS , MEDLINE and BDENF using as descriptor " Public health nursing " and the keyword " CIPESC " . The research universe consisted of 60 articles, 14 articles were nevertheless part of the sample. It was researched and comprehensive online publications between 2000 and 2012 . According to the results of the research can demonstrate that the method was descriptive and exploratory greater representability in the analyzed articles . In $72 \%$ of the article, there were no theories on the use of research by researchers. Refers to year of publication, the year 2007 showed greater prominence. However, it is noteworthy that the use of this instrument enhances nursing practices, bringing visibility and autonomy , to promote a way of thinking consciously and systematically within the practice of public health.
\end{abstract}

Keywords: Public health nursing. Rating. CIPESC. Nursing. Public Health Issues. 


\section{INTRODUÇÃO}

$\mathrm{O}$ uso do processo de enfermagem como método científico sistematizado objetivando o cuidado individualizado, focando a pessoa e não a doença, através da relação interativa do enfermeirocliente/família/comunidade, é uma forma de garantir reconhecimento profissional, visibilidade das práticas e humanização da assistência.

A utilização dos sistemas de classificação de enfermagem como instrumento das ações na sistematização da assistência de enfermagem contribui para a conscientização dos profissionais de enfermagem para a reflexão do seu trabalho tendo em vista a resolutividade, eqüidade, integralidade e qualidade da atenção à saúde nos serviços de saúde de acordo com os princípios e diretrizes do sistema único de saúde (SUS).

Sistemas de classificação são conhecimentos estruturados nos quais os elementos substantivos de uma disciplina ou subdisciplina são organizados em grupos ou classes com base em suas similaridades (BLEGEN; REIMER, 2000).

Os sistemas de classificação de enfermagem surgiram como possibilidades de contribuir para a legitimação e a oficialização das práticas dos profissionais de enfermagem. Consistindo, então, em um instrumento que favorece no gerenciamento da atenção à saúde, oferecendo à clientela uma assistência profissional, organizada, dinâmica, segura, resolutiva e competente.

Vários têm sido os sistemas de classificações existentes, entre eles existe a classificação internacional das práticas de enfermagem (CIPE). Esta foi criada na década de 1980 como um desafio de universalizar a linguagem em enfermagem, a qual fosse capaz de descrever e comunicar as atividades de enfermagem de forma clara e concisa. Portanto, durante o Congresso Quadrienal do CIE, realizado em Seul, em 1989, foi votada e aprovada a proposta para seu desenvolvimento (GARCIA; NÓBREGA, 2000).

Apesar da grande contribuição nas práticas de enfermagem e nos serviços de saúde, profissionais e estudiosos observaram que os fenômenos encontrados na CIPE eram voltados para um contexto hospitalar, deixando à parte ou pouco enfatizando a realidade da assistência extra-hospitalar ${ }^{3}$. Neste cenário, com o intuito de atender a demanda a saúde coletiva, ampliando as práticas de enfermagem no contexto do sistema único de saúde (SUS), surgiu no Brasil a Classificação Internacional das Práticas de Enfermagem em Saúde Coletiva (CIPESC). 
O projeto CIPESC foi elaborado $\mathrm{e}$ desenvolvido sob a orientação do CIE, contou com o auxílio financeiro da fundação F.W.Kellog e da ABEn, que em 1996, promoveu a primeira oficina de trabalho no Brasil que deu origem ao projeto. A ABEn é o órgão principal de disseminação do projeto através de publicações pelo seu site (ANTUNES, 2000).

$\mathrm{O}$ primeiro município do Brasil a utilizar os resultados da CIPESC como instrumento sistematizado da prática de enfermagem em saúde coletiva implementando-o no prontuário eletrônico através da rede informatizada na atenção básica municipal foi o de Curitiba em julho de 2004 (CUBAS et. al., 2006).

A CIPESC é um instrumento do processo de trabalho assistencial do enfermeiro em saúde coletiva que favorece a organização do trabalho, trazendo valorização, benefícios e impactos da ação da enfermagem tanto na assistência quanto na gerencia e na pesquisa. Portanto, é de fundamental importância conhecimentos consubstanciados nas teorias de enfermagem, pois a sua utilização orientam as práticas de enfermagem descrevendo, explicando ou prevendo os fenômenos de enfermagem (CUBAS; EGRY, 2008).

É neste contexto que surgem as questões desta revisão: Como está a produção de conhecimento acerca da classificação das práticas de enfermagem em saúde coletivaCIPESC, no Brasil?

O presente estudo teve como objetivo analisar a produção científica acerca da classificação internacional das práticas de enfermagem em saúde coletiva - CIPESC, disponível na literatura científica no período de 2000 a 2012.

\section{METODOLOGIA}

Trata-se de uma pesquisa integrativa, realizada em maio e julho de 2013, nas bases de dados disponíveis na Biblioteca Virtual em Saúde, restrita a área de ciências da saúde em geral. Foram selecionados os artigos científicos publicados no período de 2000 a 2012, relacionados a CIPESC, nas bases de dados eletrônicas em Ciências da Saúde em Geral, englobando a Scientific Electronic Library Online (SciELO), Literatura LatinoAmericana e do Caribe em Ciências da Saúde (LILACS) e Medical Literature Analysis and Retrieval System Online (MEDLINE), além da base a específica para Enfermagem: a Base de Dados de Enfermagem (BDENF), pois que o sistema de classificação em estudo é específica da profissão. Foram utilizados os descritores "Enfermagem em saúde pública", assim como a palavra-chave "CIPESC", por ser o foco desta pesquisa apesar do termo não 
estar indexado entre os Descritores em Ciências da Saúde - DeCS.

Em uma primeira busca foram identificados 60 artigos, contudo 14 artigos fizeram parte da amostra. Todas as respostas produzidas foram organizadas em um quadro sistemático, a partir das quais se procedeu à análise dos dados, permitindo a elaboração de gráficos e quadro.

\section{RESULTADOS E DISCUSÃO}

A partir das buscas de artigos nas bases de dados consultadas na biblioteca virtual em saúde, foram encontrados 14 artigos na integra que estão apresentados a seguir no gráfico 1.

Gráfico 1: Distribuição dos artigos completos sobre a CIPESC nas base de dados selecionadas para o estudo

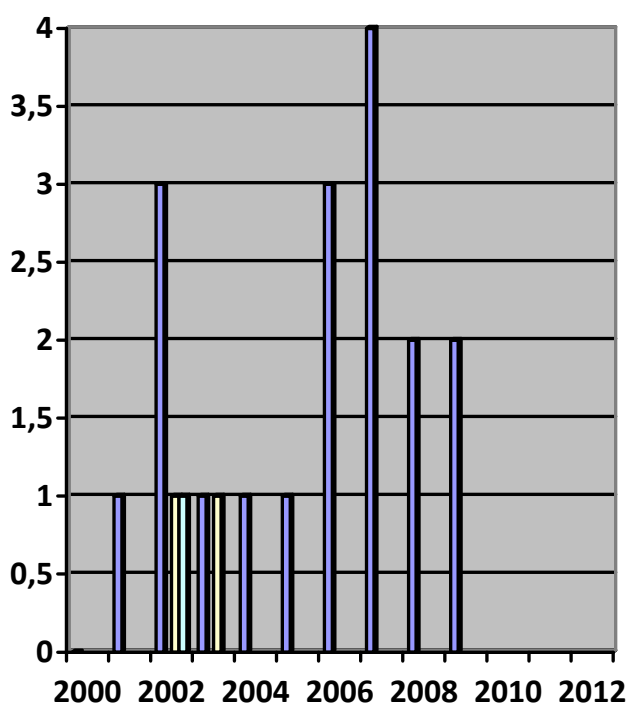

amostra, podemos observar uma maior produção ocorreu no período de 2007, esse número pode ser justificado pelo interesse dos estudiosos de avaliar a implementação do CIPESC nas práticas de enfermagem em saúde coletiva, para saber se o referente projeto tem sido utilizado como ferramenta fundamental para qualificar o trabalho de enfermagem e contribuir nos processos de intervenção de saúde e doença das populações. Entretanto, nos anos de 2010 a 2012 as publicações não fizeram parte das bases de pesquisas indexadas na biblioteca virtual de saúde. Tal fato merece uma atenção por parte dos profissionais de saúde e pesquisadores, visto que a implementação do CIPESC caracteriza-se com um instrumento que tem por finalidade nortear os profissionais em suas práticas embasadas cientificamente em teorias voltas para saúde coletiva, favorecendo a população um atendimento adequado.

De acordo com os anos de publicação dos estudos selecionados para compor a 
Quadro 1 : Tipos de estudos publicados nos artigos referente ao CIPESC

\begin{tabular}{|lr|}
\hline Tipo de pesquisa & Quantidade \\
\hline Descritivo-exploratório & 4 \\
Bibliográfica & 2 \\
Relato de experiência & 2 \\
Reflexão teórica & 2 \\
Analítica/ descritiva & 1 \\
Avaliativa documental & \\
retrospectiva & 1 \\
Descritiva documental & 1 \\
qualitativa & 1 \\
Não cita & \\
\hline
\end{tabular}

Quanto ao desenho de pesquisa dos trabalhos analisados, a maioria dos estudos utilizou o método descritivo - exploratório, que tinha como finalidade avaliar a implementação da CIPESC e suas contribuições no âmbito da saúde coletiva, bem como analisar transformações trazidas pelo projeto, contribuindo para a qualificação do trabalho em enfermagem e possibilitando para uma melhor definição das necessidades de saúde individuais e coletivas.

Nota-se que as metodologias mais utilizadas pelos artigos demonstram a contínua busca dos autores por validação da CIPESC como instrumento que aprimore a assistência de enfermagem. Uma busca que ocorreu tanto por uma perspectiva no campo da prática de enfermagem, através do método descritivo-exploratório e relato de experiência, quanto pela perspectiva de retomada da produção derivada do projeto CIPESC, através da pesquisa bibliográfica e a reflexão teórica.

O projeto CIPESC é capaz de desencadear o raciocínio clínico, julgamento e a tomada de decisão do enfermeiro por meio do diagnostico de enfermagem e o plano de cuidado, nos espaços extra - hospitalares, transformando o trabalho em saúde, comprometido com os princípios da reforma sanitária do Sistema Único de Saúde (SUS), visando a integralidade da assistência (ANTUNES, 2000).

Observou-se que a maioria dos estudos analisados foram desenvolvidos nos municípios de Paraná, Curitiba, Porto Alegre e João Pessoa, provavelmente como reflexo da implantação do CIPESC ocorrer de forma pioneira na regiao Sul do Brasil. Tal quadro demonstra a importância do desenvolvimento do projeto em demais regiões do país (ALTINO et al., 2006; SANTOS; NÓBREGA, 2004). 
Gráfico 2: Teorias utilizadas nos artigos publicados referente aos CIPESC

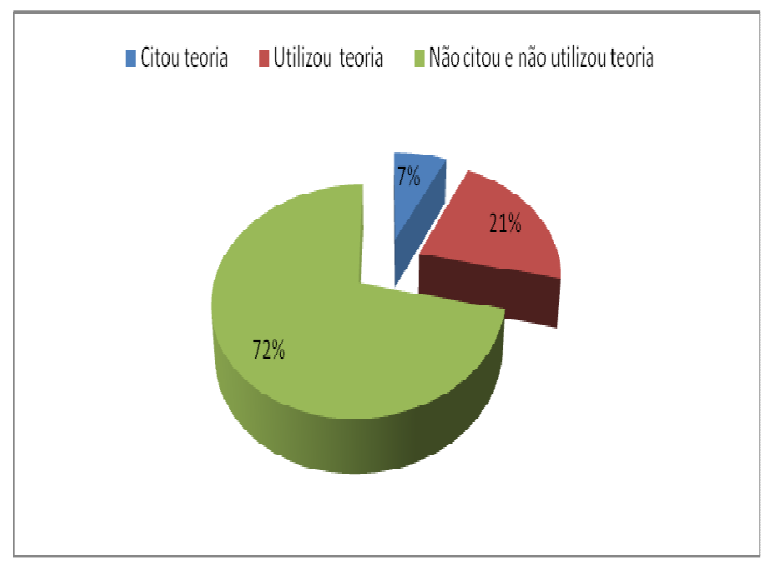

No gráfico 2 observou-se $21 \%$ (3) dos artigos analisados utilizaram teoria em seus estudos, estes dados demonstra que as teorias pode ser aplicada nas práticas dos profissionais de enfermagem, favorecendo para uma melhor definição de seu trabalho e operacionalização das ações.

As teorias compreendem um conjunto de conceitos e pressupostos, relacionados entre si, compreendendo o campo da prática, do ensino e da pesquisa. Tais teorias referemse ao saber - fazer, fornecem bases para prática, trazendo autonomia e identidade a profissão (LEOPARDI, 2006).

Entretanto, a maioria das publicações $72 \%$ (10), como visto no gráfico 2 , não mencionou a utilização das teorias. Este dado pode está relacionado ao fato de que a escolha de uma teoria de enfermagem para guiar a prática, não é fácil, tendo em vista que requer conhecimentos destas e dos fenômenos das situações clínicas do indivíduo ou da comunidade a quem se presta o cuidar (GARCIA; NOBREGA, 2010).

Diversos autores mencionam a dificuldade dos enfermeiros na prática em implantar o CIPESC como decorrente da graduação, justificando que houve pouca familiaridade com os sistemas de classificação de enfermagem e sistemas informatizados. Enfatizando a necessidade de programas de educação contínua para os enfermeiros para consolidação e expansão do CIPESC (CUBAS, LOPES, VAZ, 2006; CUBAS, 2009)

Quanto às contribuições do CIPESC, Percebe-se uma influência histórica do foco da prática de saúde guiada pelo modelo biomédico, focalizando a assitência no indivíduo e na doença, sendo detectado por diversos autores a necessidade do foco ser voltado ao processo saúde doença, integrando o ambiente com a sociedade e contribuindo à integralidade da assistência a saúde. Nota-se, então, como a CIPESC incentiva esta mudança nos paradigmas da assistência, ao mesmo tempo em que o contextualiza com os determinantes e as necessidades sociais do paciente (CUBAS; EGRY, 2008) .

Entretanto, observa-se a necessidade de embasamento da prática profissional em teorias de enfermagem por refletirem no reconhecimento da Sistematização da Assistência de enfermagem e, 
conquentemente, na autonomia do enfermeiro em sua atuação profissional, por haver uma retomada das atribuições específicas desta categoria profissional como a elaboração do Processo de Enfermagem. Contudo, há necessidade dos enfermeiros desenvolverem uma prática profissional consciente e reflexiva para usufruir das contribuições do CIPESC tanto na otimização do cuidado de enfermagem quanto como um instrumento facilitador da avaliação da prática da equipe de enfermagem. (BARROS; CHIESA, 2007)

Além disto, diversos autores descrevem a contribuição do projeto às ações de enfermagem por possibilitar sistematização da assistência captando de forma específica às necessidades de saúde de grupos específicos, como gestantes, crianças e portadores da Síndrome da Imunodeficiência Humana Adquirida - SIDA, o que possibilita a flexibilidade de implantação do CIPESC aos diversos usuários do sistemA (SILVA et al., 2010).

A vantagem da implantação de um prontuário eletrônico da CIPESC para sistematizar a consulta de enfermagem é reconhecida por diversos autores por ser um instrumento que facilita a comunicação e padronização das práticas, tanto em âmbito nacional quanto internacional (ALTINO et al.,2006). Enfatiza-se, então, a importância da validação contínua dos termos mantendo a assistência adequada à realidade, havendo ainda necessidade de expansão da nomenclatura para ampliar as áreas de atenção à saúde do CIPESC, tendo em vista a agillização dos processos de avaliação e fortalecimento da prática (SILVA et al., 2010; APOSTOLICO et al., 2007)

\section{CONSIDERAÇÕES FINAIS}

Diante do atual contexto e considerando a divulgação dos resultados das pesquisas referente ao projeto CIPESC, observa-se uma produção do conhecimento bastante escasso nos bancos de dados analisados. Isto ocorre por ainda estar em processo de reconhecimento e implementação pelos profissionais e instituições de saúde no âmbito coletivo, citado em diversos estudos. Apesar de apenas alguns artigos serem disponibilizados completos e online acerca do projeto, ainda assim os enfermeiros conseguem obter uma perspectiva geral da validação do projeto devido às metodologias frequentemente utilizadas nestes estudos. Portanto, a disseminação do conhecimento sobre este é de bastante relevância já que seu conhecimento e sua utilização possibilitam o aprimoramento das práticas de enfermagem, conforme destacado por diversos artigos do estudo. 
Referente às citações das teorias, poucos estudos abordaram em sua essência metodológica um referencial teórico. Isto demonstra uma fragilidade da produção de conhecimento, pois as teorias têm contribuído no campo da prática profissional (ensino, pesquisa ou assistência), orientando as ações e favorecendo um modo de pensar sistemático sobre a enfermagem e suas intervenções.

Os sistemas de classificação apresentam limitações, como por exemplo a mecanização e fragmentação das práticas de trabalho, sendo necessária a compreensão reflexiva dos profissionais acerca da assistência voltada ao cuidado integral. Assim como tornar pública as nossas práticas, desenvolvendo a visibilidade política da enfermagem.

Com o intuito de aprimorar e ampliar o estudo e contribuições do Projeto CIPESC, assim como das teorias de enfermagem, há necessidade de desenvolver estratégias no Brasil que englobem, inclusive, a formação dos enfermeiros. Buscando incentivar jovens criativos, estimulando-os a integrar os grupos de pesquisa com um objetivo comum: atender as demandas da sociedade. Desenvolvendo, assim, um processo de reflexão sobre o cuidado diário de enfermagem voltado tanto a atenção em saúde individual quanto coletiva.

Com esses resultados, pode-se caracterizar como têm sido conduzidas as investigações sobre o projeto CIPESC nos bancos de dados citados no estudo. Logo, através do estímulo da reflexão e construção de conhecimento, busca-se contribuir nas áreas de ensino e pesquisa com o intuito de incentivar a disseminação de informações que possibilite intervenções qualificadas na prática de saúde coletiva. Desta forma, a CIPESC consiste em um importante recurso que o enfermeiro dispõe para aplicar e demonstrar seus conhecimentos científicos, técnicos e humanos no cuidado ao paciente e caracterizar dessa forma, sua prática profissional.

\section{REFERÊNCIAS}

\section{ALTINO, D. M. et al. CIPESC® Curitiba: o} trabalho da enfermagem no Distrito Bairro Novo. Rev. bras. enferm. v. 59, n.4, 2006.

ANTUNES, M. J. M. A prática de enfermagem e os sistemas de classificação: a ótica da ABEn. In: Garcia Telma Ribeiro; Nobrega Maria Miriam Lima da. Sistemas de classificação da prática de enfermagem: um trabalho coletivo. João Pessoa: Idéia, 2000.

APOSTOLICO, M. R. et. al.Contribuição da CIPESC® na execução das políticas de atenção à saúde da criança no município de 
Curitiba, Paraná. Texto contexto - enferm. v.16, n.3, 2007.

BARROS, D. G.; CHIESA, A. M. Autonomia e necessidades de saúde na sistematização da assistência de Enfermagem no olhar da saúde coletiva; v. 41,2007.

BLEGEN, M. A.; REIMER, T. T.

Implications of Nursing Taxonomies for

Middle-RangeTheory Development. In:

NOBREGA, M. M. L. da; GUTIERREZ, M.

G. R. de. Sistemas de Classificação na

Enfermagem: avanços e perspectivas.

Sistemas de Classificação em Enfermagem: um trabalho coletivo. João Pessoa, Idéias, 2000.

CUBAS, M. R. et al. Sistematizando a prática de enfermagem na SMS Curitiba. In: Altino Denise Maria, Apostolico Maíra Rosa, Duarte Franciele de Oliveira, Cubas Márcia Regina, Egry Emiko Yoshikawa. CIPESC® Curitiba: o trabalho da enfermagem no Distrito Bairro Novo. Rev. bras. enferm. v. 59, n.4, 2006.

CUBAS, M. R. Instrumentos de inovação tecnológica e política no trabalho em saúde e em enfermagem: a experiência da CIPE®/CIPESC®.; v. 62, n.5, 2009.
CUBAS, M. R.; EGRY, E. Y. Classificação Internacional de Práticas de Enfermagem em Saúde Coletiva - CIPESC®. Rev. esc.

enferm. USP, v. 42, n.1 2008.

CUBAS, M. R.; EGRY, E. Y. Classificação Internacional de Práticas de Enfermagem em Saúde Coletiva - CIPESC®. Rev. esc.

enferm. USP [serial on the Internet]. 2008

Mar [cited 2010 June 8] ; 42(1): 181-186.

GARCIA, T. R.; NOBREGA, M. M. L. da.

Sistemas de classificação da prática de enfermagem: um trabalho coletivo. João

Pessoa: Idéia, 2000.

\section{LEOPARDI, M. T. Teoria e Método em}

Assistência de Enfermagem. 2. Ed. rev.

Ampl. Florianópolis: Ed. Soldasoft, 2006.

SANTOS, S. M. J. dos; NÓBREGA, M. M.

L. da. Ações de enfermagem identificadas no Projeto CIPESC(a) e utilizadas no cuidado de pacientes com AIDS.; v. 38, n.4, 2004.

SILVA et al. Estudo avaliativo da consulta de enfermagem na Rede Básica de Curitiba PR; v.44, n.1, 2010. 